\title{
RESOLVING THE CONTROVERSY OVER TIN AND GALLIUM MELTING IN A RECTANGULAR CAVITY HEATED FROM THE SIDE
}

\author{
Noureddine Hannoun \\ Department of Mathematics, University of Tennessee, Knoxville, \\ Tennessee, USA \\ Vasilios Alexiades \\ Department of Mathematics, University of Tennessee, Knoxville, Tennessee, \\ USA, and Oak Ridge National Laboratory, \\ Oak Ridge, Tennessee, USA

\section{Tsun Zee Mai} \\ Mathematics Department, University of Alabama, Tuscaloosa, Alabama, USA
}

\begin{abstract}
"Is the flow monocellular or multicellular?"' This question was first raised by Dantzig 13 years ago. It was about the nature of the fluid flow for the gallium melting problem in a rectangular cavity heated from the side. Following Dantzig's work, several publications have appeared on the same problem, with fewer than a handfull reporting a multicellular structure. To date, all experimental results support the monocellular structure, yet some researchers claim that the monocellular solution is incorrect. A similar controversy was reported for the problem of tin melting, suggested in 1998 as a benchmark problem. Several arguments have been suggested to explain the discrepancies, but there does not seem to be a commonly accepted answer to the problem in the scientific community. In this work, we summarize earlier works and present a grid-refinement study for several discretization schemes with emphasis on tin melting and some results for gallium melting. Simulations are carried out with the enthalpy method. The flow cell structure is analyzed in detail, while some results are provided for the heat transfer and the melting rate. Our results show that the multicellular structure is the correct numerical solution and that the flow structure has a strong influence on other features of the solution. We also provide a detailed discussion of earlier results in order to clarify important issues and bring a final answer to the controversy.
\end{abstract}

Received 15 November 2002; accepted 8 March 2003.

The authors wish to thank Dr. D. Gobin for making available some of the earlier tin melting results. Access to the Compaq AlphaServer SC computer was provided by the Center for Computational Sciences at Oak Ridge National Laboratory and the Evaluation of Early Systems research project, sponsored by the Office of Mathematical, Information, and Computational Sciences, Office of Science, U.S. Department of Energy. Oak Ridge National Laboratory is managed by UT-Battelle, LLC, for the U.S. Department of Energy under Contract DE-AC05-00OR22725. The U.S. government retains a nonexclusive, royalty-free license to publish or reproduce the published form of this contribution, or allow others to do so, for U.S. government purposes. Access to the Cray SV1 supercomputer was provided by the Alabama Supercomputer Authority, which provides supercomputing time free of charge to faculty and students of the state-funded schools of Alabama.

Address correspondence to Noureddine Hannoun, University of Tennessee, Department of Mathematics, Knoxville, TN 37996-1300, USA. E-mail: hannoun@math.utk.edu 


\begin{tabular}{|llll|}
\hline \multicolumn{3}{c|}{ NOMENCLATURE } \\
$a_{P}$ & central node coefficient & $v$ & y-velocity component \\
$a_{P}^{o}$ & old time coefficient & $\vec{V}$ & velocity vector \\
$A$ & porosity constant & $W$ & cavity width \\
$\mathrm{Ar}$ & cavity aspect ratio $(=H / W)$ & $x, y$ & Cartesian coordinates \\
$c_{p}$ & specific heat & $\alpha$ & thermal diffusivity $\left(=\kappa / \rho c_{p}\right)$ \\
$C$ & porosity constant & $\beta$ & coefficient of thermal expansion \\
$f_{L}$ & liquid fraction & $\Delta H$ & latent enthalpy content \\
$g$ & gravity acceleration & $\epsilon$ & numerical solidification $T$ range \\
$h$ & sensible enthalpy $\left(=c_{p} T\right)$ & $\epsilon_{o}$ & outer iterations convergence tolerance \\
$H$ & cavity height & $\kappa$ & thermal conductivity \\
$L$ & Latent heat of fusion & $\mu$ & dynamic viscosity \\
$\mathrm{Nu}$ & Nusselt number $\left(=Q_{w} / Q_{c}\right)$ & $v$ & kinematic viscosity \\
$P$ & pressure & $\rho$ & density \\
$\mathrm{Pr}$ & Prandtl number $(=v / \alpha)$ & $\psi_{\text {min }}$ & minimum streamfunction value \\
$q$ & porosity constant & $\omega$ & underrelaxation parameter \\
$Q_{c}$ & total heat flux without convection & & \\
$Q_{w}$ & heat flux through the hot wall & Subscripts \\
$\mathrm{Ra}$ & Rayleigh number & $c$ & cold wall \\
& [=g $\left.\beta\left(T_{h}-T_{f}\right) H^{3} / \alpha v\right]$ & $f$ & melting or freezing \\
$\mathrm{St}$ & Stefan number $\left[=c_{p}\left(T_{h}-T_{c}\right) / L\right]$ & $h$ & hot wall \\
$t$ & time & $L$ & liquidus \\
$T$ & temperature & ref & reference value at $T_{f}$ \\
$u$ & x-velocity component & $S$ & solidus \\
& & & \\
& & & \\
\hline
\end{tabular}

\section{INTRODUCTION}

Phase-change problems often involve nonlinearities, strong couplings, and a moving boundary [1]. As a result, only the simplest configurations are amenable to analytical tools, while solving most problems of interest requires the use of numerical methods. An additional difficulty is introduced due to the necessity of assessing the accuracy of the numerical solution. This is done by testing the code on test problems with known solutions, called benchmarks problems. Neither analytical solutions nor benchmark solutions are currently available for phase-change problems involving convection in the melt. Hence, the assessment of numerical solutions in past studies was done by comparison with experimental results or other numerical results, usually obtained by alternative numerical techniques.

The problem of gallium or tin melting in a rectangular cavity heated from the side has been used extensively by researchers for the assessment of phase-change numerical methods. Indeed, when convection in the melt is involved, this is one of the few problems available in the literature for comparison purposes. Gallium melting has been studied both numerically [2-25] and experimentally [26, 27]. Several distinct configurations have actually been considered. Cavity aspect ratio values $\mathrm{Ar}=1[6,10-13], \mathrm{Ar}=0.714$ (the most common) $[2,3,6,7,14,16,18-20,22-27]$, $\mathrm{Ar}=0.5[4,6,7,9,15,17,21,24,27-29]$, and $\mathrm{Ar}=0.286[24,26,27]$ have been investigated. Moreover, some authors have considered several values of the Rayleigh number, Ra, and Stefan number, St, for a given aspect ratio choice. The most 
frequent sets of parameters are $\mathrm{Ra}=6 \times 10^{5}$ and $\mathrm{St}=0.04$ for $\mathrm{Ar}=0.714$, $\mathrm{Ra}=2.2 \times 10^{5}$ and $\mathrm{St}=0.04$ for $\mathrm{Ar}=0.5$, and $\mathrm{Ra}=4 \times 10^{4}$ and $\mathrm{St}=0.04$ for $\mathrm{Ar}=0.286$. For tin melting, both experimental [30] and numerical [5, 23, 30-33] results are available. Most studies for tin melting have focused on the configuration with $\mathrm{Ar}=0.75, \mathrm{Ra}=1.2 \times 10^{5}, \mathrm{St}=0.0085$, and $\mathrm{Pr}=0.016$. Two other choices of values for $\mathrm{Ra}$ have been considered in the experimental work of Wolff et al. [30], while the aspect ratio $\mathrm{Ar}=1$ has been investigated numerically in [23,31]. More recently, Lequere and Gobin [34] promoted tin melting as a benchmark exercise. They suggested two sets of parameter values slightly different from those used in earlier studies, and called for contributions in order to perform a comparison among independent results and ultimately obtain a reference solution. The exercise, which resulted in two conference presentations and two publications [35, 36], has not yielded a reference solution yet. The tin melting configuration with $\mathrm{Ar}=1$, $\mathrm{Ra}=2.5 \times 10^{5}, \mathrm{St}=0.01$, and $\mathrm{Pr}=0.04$ is very similar to the gallium melting problem with $\mathrm{Ar}=0.7$.

Although the problems of gallium and tin melting have been employed extensively for comparison purposes, the accuracy of past results has never been clearly demonstrated. This problem has actually been offset by a controversy about the flow structure in the melt. How could researchers focus on the accuracy of results if they could not even agree on the global features of the solution? Here, the term "global features" refers to the number of cells observed in the fluid flow during the melting process. The controversy originated from the work of Dantzig [3], who found in his work on gallium melting a melt flow structure with several rolls. Prior to Dantzig's work, only one-roll patterns had been reported [2]. Moreover, the findings of Dantzig were apparently in contradiction with experimental results [27], while those of Brent et al. [2] seemed to match experiments rather well. Dantzig's findings led him to argue: "The most interesting point to pursue is the difference in the nature of the flow found in the gallium melting problem," and open up the controversy with his question: "Is the multiple cell solution or the single cell solution correct?" Dantzig, who used the commercial code FIDAP, argued that upwinding and too coarse grids were the reasons for the failure of earlier studies in predicting a multicellular flow structure. Dantzig showed that, early in the melting process, the use of upwinding for convective terms resulted in the suppression of eddies which were observed with a centered scheme. Dantzig did not succeed in supporting his second argument, for his code failed to converge when coarser grids were employed.

Following the work of Dantzig [3], numerous publications [6, 7, 14, 16, 18, 22] reported results consistent with the findings of Brent et al. [2], i.e., a single cell structure. In 1994, Campbell and Koster [26] performed experiments in order to check earlier results from Gau et al. [27], which were criticized for lack of welldefined setup. Although their results did not agree very well with those of Gau et al. (and actually brought up another problem, which they attributed to a possible density anomaly), their findings seemed to confirm that a single-roll structure was the right solution for the problem of gallium melting. The findings of Dantzig were recently confirmed by the work of Stella and Giangi [19], who used very fine grids (several times finer than in earlier studies) along with the centered discretization scheme. In an attempt to explain the disagreement of earlier numerical results with experimetnal resuls of Gau et al., Cerimele, Mansutti, and Pistella [20] suggested that 
numerical simulations performed for the gallium melting problem were not consistent with the experimental procedure, which involved a pour-in/pour-out procedure that resulted in an actual restart of the fluid in the melt from uniform temperature and zero fluid velocity. This fact was not taken into account in earlier numerical studies. Later, Cruchaga and Calentano [24] performed numerical simulations showing two recirculations early during the melting process and three later. This confirmed earlier results by Dantzig, but the number of rolls obtained was different. More recently, Wintruff and Gunther [25] presented results which match very well those of Stella et al. and indicate an initial roll structure with four rolls, but they did not provide details for the numerical parameters.

Prior to the benchmark exercise suggested by Lequere et al. [34], all results for tin melting simulations were in rather close agreement. In contrast, the newly suggested configuration resulted in the very same controversial observation as for gallium melting. However, the discrepancies were among numerical results, since no experimental results were available. The contributors to this benchmark exercise were divided into two groups. Contributors in the first group found a single-roll solution, while the second group reported a two-roll solution. Discrepancies in $\mathrm{Nu}$ plots were also reported: sudden drops and oscillations were observed in results with several-roll patterns. In their first publication [35], the contributors to the benchmark exercise argued that a reference solution for the second test problem was probably out of reach for some time, due to an unexpected instability. They also suggested that difficulties in satisfying experimentally hypotheses accepted by numerical simulations could explain discrepancies between numerics and experiments. In their last article [36], they concluded that the problem was in need of further investigation, emphasizing the need for a full description of the physical phenomena, verification of codes, grid-refinement studies, and better experiments.

In past studies about tin or gallium melting, many authors claimed their solutions were grid-converged, but few actually reported results for the grid-refinement study. Dantzig [3] failed to obtain results with coarse grids. Viswanath et al. [7] performed a refinement from a $30 \times 30$ grid to a $50 \times 50$ grid. They indicated at $3 \%$ change in main flow values and concluded that their solution was satisfactory. Similar arguments were presented by Rady and Mohanty [12], who refined the grid from $20 \times 20$ to $35 \times 35$. To the authors' knowledge, the only published refinement study is the one of Stella et al. [19]. They called their study a mesh sensitivity analysis because they did not actually try to obtain a grid-converged solution but rather limited their analysis to determining a grid that would capture the correct number of rolls in the liquid. Moreover, their results from various grids differed only slightly.

Comparisons among discretization schemes have been reported in three publications. Dantzig [3] switched from the centered scheme to first-order upwind and showed that, early in the melting process, eddies were suppressed. However, it is rather surprising that he was able to find as many as six rolls early in the melting process with the coarse grid he used; Stella et al. [19] found only four rolls (almost five) with the same scheme and a grid five times finer. Shyy et al. [13], who actually focused on gallium melting with $\mathrm{Ar}=0.5$ (not the focus of the present study), compared first- and second-order upwind schemes, and showed that there is an increase in number of rolls when the order of the discretization scheme is increased. More recently, Kim et al. [23] presented a comparison of four discretization schemes 
(upwind, hybrid, centered, power law) for the gallium melting problem. Due to their use of too coarse grids, there were no significant differences among the results from the various schemes, all showing a single roll.

The main points of the controversy about tin and gallium melting problem may be summarized as follows.

1. Contributors who found a multicellular flow pattern generally used finer grids and/or higher-order discretization schemes, while those who found a single-cell pattern generally used coarse grids and/or lower-order discretization schemes.

2. Coarse-grid and lower-order-scheme solutions agree better with experimental results than fine-grid and higher-order discretization solutions.

3. There is no unanimously accepted explanation for the disagreement between higher-order solutions and experimental observation.

4. No convergence study has been performed, although one gridrefinement study is available for gallium.

5. No clear comparison among various discretization schemes is available.

The present work aims at providing results that clearly show what the correct solutions to the gallium and tin melting problems are, while providing explanations and arguments for erroneous earlier interpretations. As a result, future directions for the problem considered are clarified.

\section{PHYSICAL PROBLEM}

Two problems are considered in the present study: the configuration for gallium melting of Brent et al. [2] and the second test problem of Lequere et al. [34] for tin melting. The general configuration is shown in Figure 1 and the flow parameters are given in Table 1 for both gallium and tin. The cold boundary is undercooled for gallium $\left(T_{c}<T_{f}\right)$ and at melting temperature for tin $\left(T_{c}=T_{f}\right)$. In both problems, the

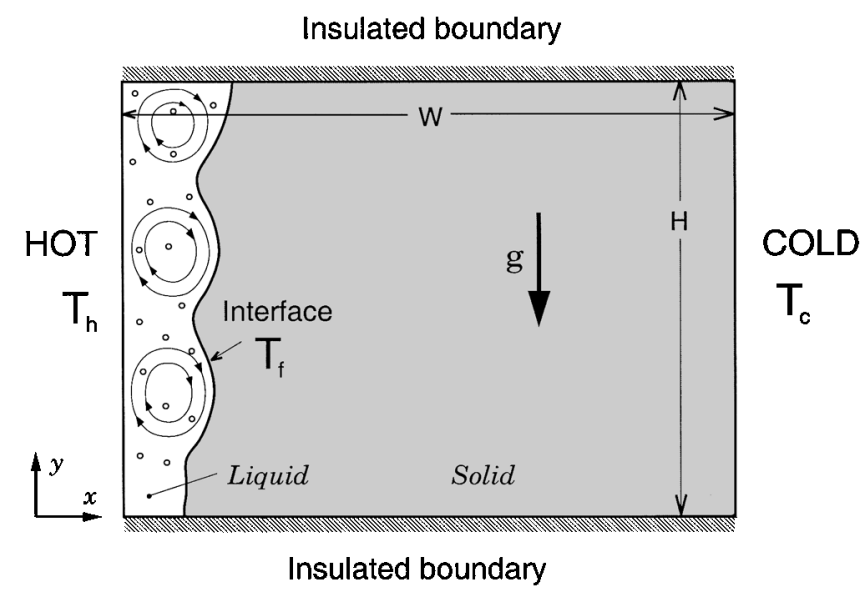

Figure 1. Tin and gallium melting problems configuration. 
Table 1. Tin and gallium parameters for melting problem

\begin{tabular}{|c|c|c|c|c|}
\hline Parameter & Symbol & Tin & Gallium & Units \\
\hline Reference density & $\rho_{\text {ref }}$ & $7.5 \times 10^{3}$ & $6.093 \times 10^{3}$ & $\mathrm{~kg} / \mathrm{m}^{3}$ \\
\hline Specific heat & $c_{\mathrm{p}}$ & 200 . & 381.5 & $\mathrm{~J} / \mathrm{kg} \mathrm{K}$ \\
\hline Dynamic viscosity & $\mu$ & $6 . \times 10^{-3}$ & $1.81 \times 10^{-3}$ & $\mathrm{Ns} / \mathrm{m}^{2}$ \\
\hline Thermal conductivity & $\kappa$ & 60 & 32. & $\mathrm{~W} / \mathrm{m} \mathrm{K}$ \\
\hline Coefficient of volume expansion & $\beta$ & $2.67 \times 10^{-4}$ & $1.20 \times 10^{-4}$ & $\mathrm{~K}^{-1}$ \\
\hline Latent heat of fusion & $L$ & 6. $\times 10^{5}$ & $8.016 \times 10^{4}$ & $\mathrm{~J} / \mathrm{kg}$ \\
\hline Fusion temperature & $T_{f}$ & 505. & 302.78 & $\mathrm{~K}$ \\
\hline Hot wall temperature & $T_{h}$ & 508. & 311. & $\mathrm{~K}$ \\
\hline Cold wall temperature & $T_{c}$ & 505. & 301.3 & $\mathrm{~K}$ \\
\hline Cavity height & $H$ & 0.1 & 0.0635 & $\mathrm{~m}$ \\
\hline Cavity width & $W$ & 0.1 & 0.0889 & $\mathrm{~m}$ \\
\hline Cavity aspect ratio $(H / W)$ & $A r$ & 1. & 0.714 & \\
\hline Gravity acceleration & $g$ & 10. & 10. & $\mathrm{~m} / \mathrm{s}^{2}$ \\
\hline Rayleigh number & $\mathrm{Ra}$ & $2.25 \times 10^{5}$ & 7. $\times 10^{5}$ & \\
\hline Stefan number & $\mathrm{St}$ & 0.01 & 0.046 & \\
\hline Prandtl number & $\operatorname{Pr}$ & 0.04 & 0.0216 & \\
\hline
\end{tabular}

material in the cavity is initially solid at temperature $T_{c}$. The temperature of the left wall is suddenly raised to $T_{h}$ at time $t=0$, and melting of the solid starts. Convection in the melt results in one or several recirculations.

\section{MATHEMATICAL MODEL}

The basic formulation of our mathematical model is that of Brent et al. [2]. The model assumes the fluid to be a porous medium with a porosity undergoing a sharp change at the melting temperature. The setting is two-dimensional, incompressible, unsteady, and laminar. The fluid, a single-component metal, is Newtonian, and has constant thermophysical properties throughout the temperature range of study. Buoyancy effects are dealt with the Boussinesq approximation. This results in the model Eqs. (1).

$$
\begin{gathered}
\frac{\partial \rho}{\partial t}+\nabla \cdot\left(\rho \nabla_{\vec{V}}\right)=0 \\
\frac{\partial \rho u}{\partial t}+\nabla \cdot(\rho \vec{V} u)=\nabla \cdot(\mu \nabla u)-\frac{\partial P}{\partial x}-A u \\
\frac{\partial \rho v}{\partial t}+\nabla \cdot(\rho \vec{V} v)=\nabla \cdot(\mu \nabla v)-\frac{\partial P}{\partial y}-A v+\frac{\rho_{\mathrm{ref}} g \beta\left(h-h_{\mathrm{ref}}\right)}{c_{p}} \\
\frac{\partial \rho h}{\partial t}+\nabla \cdot(\rho \vec{V} h)=\nabla \cdot\left(\frac{\kappa}{c_{p}} \nabla h\right)-\frac{\partial \rho \Delta H}{\partial t}-\nabla \cdot(\rho \vec{V} \Delta H)
\end{gathered}
$$

The constant $A$ in the source terms of the momentum equations $(1 b)-(1 c)$ is provided by Eq. (2), where $C$ and $q$ are two constants whose values depend on the problem considered. 


$$
A=-\frac{C\left(1-f_{L}\right)^{2}}{f_{L}^{3}+q}
$$

In the mushy zone $T_{S} \leq T \leq T_{L}$, the liquid fraction $f_{L}$ is related to temperature $T$ through a linear relationship, with values ranging from 0 in the solid to 1 in the liquid.

The mathematical model is complemented with appropriate initial and boundary conditions. On the boundary, both velocity components are set to zero. Both top and bottom boundaries are assumed to be thermally insulated, while the temperature is specified on the lateral walls. Numerical simulations are started from fluid at rest and at uniform temperature $T=T_{c}$.

\section{NUMERICAL METHOD AND PARAMETERS}

The conservation equations (1) are discretized by a finite-volume method on a uniform Cartesian grid. Approximations for the various terms are second-orderaccurate. However, the convective terms may be handled by any of the following five schemes: upwind, hybrid, centered, power law, and exponential. A fully implicit Euler method is employed for time discretization. The coupling energy-liquid fraction, resulting from the use of an enthalpy formulation, is handled by the procedure suggested in [2]. The resulting nonlinear and coupled system of equations is handled by the SIMPLER algorithm. Linear systems are solved by two iterative methods: BICGSTAB-SIP for nonsymmetric systems, and CG-SSIP for symmetric systems.

For both tin and gallium melting, the flow equations are solved in a reduced domain containing the liquid. This practice is also used with the energy equation for tin melting, since the solid is isothermal. The numerical melting range is chosen as follows: for gallium melting, $T_{L}=T_{f}+\epsilon / 2$ and $T_{S}=T_{f}-\epsilon / 2$; while for tin melting, $T_{L}=T_{f}+\epsilon$ and $T_{S}=T_{f}$.

The simulations are carried out with the following numerical parameter values: $\epsilon=0.025, \Delta t=0.1$ to $0.01 \mathrm{~s}$ (decreases as melting proceeds), $C=10^{15}, q=10^{-6}$, $\omega_{\Delta H}=0.2, \omega_{u}=\omega_{v}=0.7, \omega_{P}=0.9$, and $\omega_{h}=0.8$. Inner iterations (for linear systems) are stopped when the residual is reduced by a factor $10^{7}$, while outer iterations are stopped when the residual of the conservation equation is less than $\epsilon_{0}$, where $\epsilon_{0}$ is set to $10^{-2}$ for momentum and energy, and $10^{-5}$ for mass in the case of gallium, and $8 \times 10^{-2}$, $10^{-1}$, and $10^{-5}$ for momentum, energy, and mass, respectively in the case of tin.

The code used for the present study has been thoroughly verified on a series of test problems. Each component of the code has been checked separately. A final test problem has been used to assess the good functioning of the coupling among the various solvers. Details of the verification procedure may be found in [37].

\section{RESULTS FOR TIN}

\subsection{Streamlines and Roll Pattern}

Figures 2-4 display the streamlines and solid-liquid interface obtained at several times during the melting process. Three discretization schemes (upwind, hybrid, and centered) and three grids $(100 \times 100,200 \times 200$, and $400 \times 400)$ are 


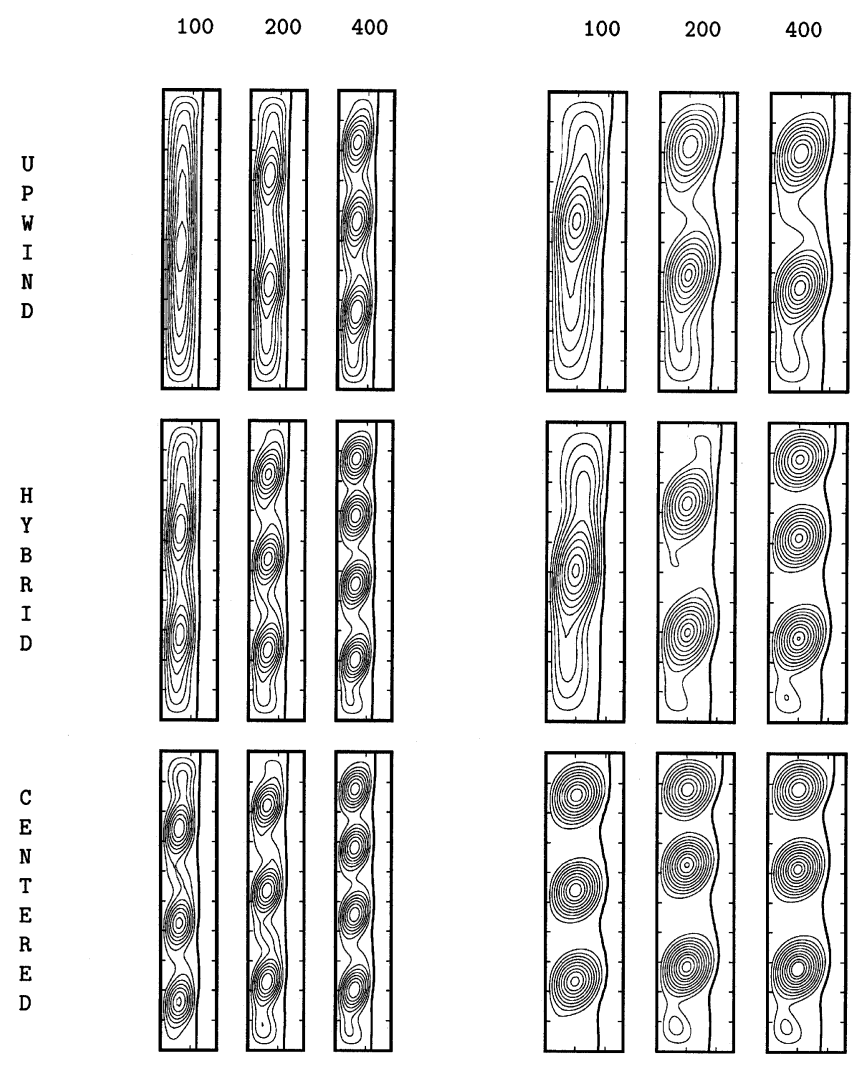
(a) $t=200 \mathrm{~s}$
(b) $t=450 \mathrm{~s}$

Figure 2. Streamlines and interface at times $200 \mathrm{~s}$ and $450 \mathrm{~s}$ for three grids and three discretization schemes.

considered for the comparison. Each time corresponds to a set of nine pictures, with each scheme corresponding to a row and each grid size to a column. Numerically, the least accurate of the nine pictures at one particular time is the top left, while the most accurate is the bottom right. The left boundary of each picture corresponds to the left hot wall, while the top and bottom boundaries represent the isothermal walls. The isothermal solid to the right of the solid liquid interface has been truncated to allow the pictures to fit in a single page. The spacing between two major tickmarks in each direction corresponds to one-tenth of the total height (width). The solid-liquid interface is determined by the liquid fraction contour $f_{L}=0.5$.

Several important conclusions may be drawn from Figures 2-4. First, the number of rolls increases as the grid is refined. Although most plots convey this observation quite well, this is better exemplified by the three plots obtained at time $200 \mathrm{~s}$ with the hybrid scheme: the number of rolls is seen to increase from two to four as the grid is refined. Similarly, at time $450 \mathrm{~s}$ for the same scheme (hybrid), the number of rolls increases from one to three as the grid is refined. Second, the number of rolls increases as the order of the discretization scheme increases. This is best shown 


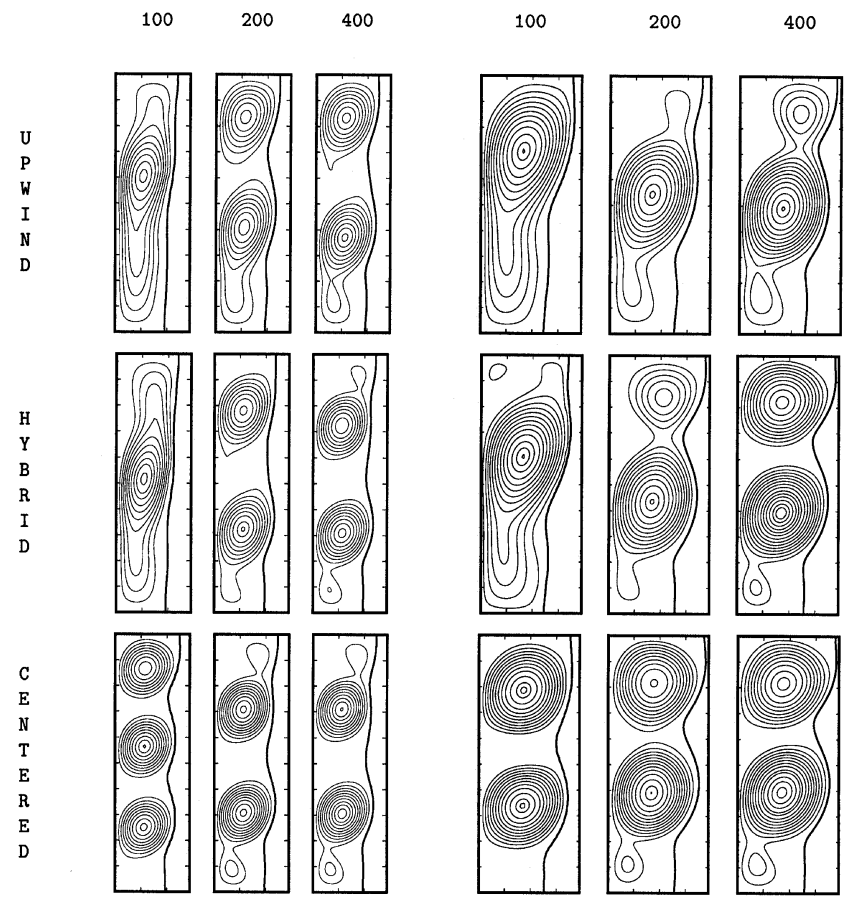

$\begin{array}{ll}\text { (a) } t=550 \mathrm{~s} & \text { (b) } t=1000 \mathrm{~s}\end{array}$

Figure 3. Streamlines and interface at times $550 \mathrm{~s}$ and $1,000 \mathrm{~s}$ for three grids and three discretization schemes.

on the plots obtained at time $200 \mathrm{~s}$ with the $100 \times 100$ grid: the number of rolls obtained is one for upwind, two for hybrid, and three for centered. Third, the discrepancy among the results obtained by the three schemes and the three grids decreases with time, as all of them end up with one single roll at time 2,000 s. However, the single cell is located farther down in the cavity for finer grids and for higher-order schemes. This indicates that melting is faster at the bottom and slower at the top for more accurate solutions. Fourth, the rolls are well rounded for higher-order schemes and flat for lower-order schemes, as may be seen on the plots obtained at time $550 \mathrm{~s}$, for example.

Also notice that the number of rolls obtained with the centered scheme does not change significantly when the grid is refined. This indicates that the solution is close to grid-converged, a good hint to undertake a grid convergence study. The largest difference in roll number at a given time is expected to be between the top left (upwind $-100 \times 100$ ) and the bottom right (centered $-400 \times 400$ ) plots. Notice that the difference decreases with time. At time $200 \mathrm{~s}$, the most accurate solution shows four rolls and the least accurate has one roll. We use the notation (4:1) to refer to this fact. Then, at time $450 \mathrm{~s}$, the roll contrast is (3:1), at time $1,000 \mathrm{~s}(2: 1)$, and at time 2,000 s (1:1).

As a general rule, we may conclude that more accurate solutions exhibit a larger number of rolls. In particular, the number of rolls is greater for finer grids. This rule, 
100
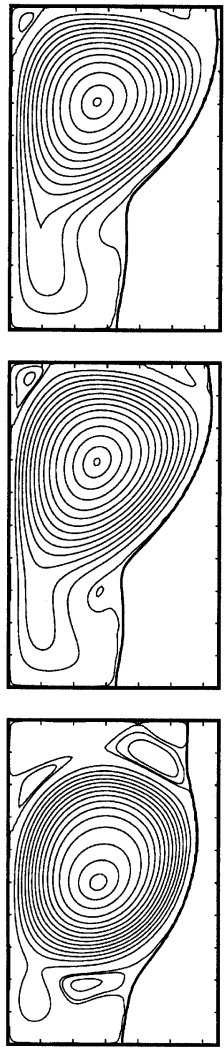

200
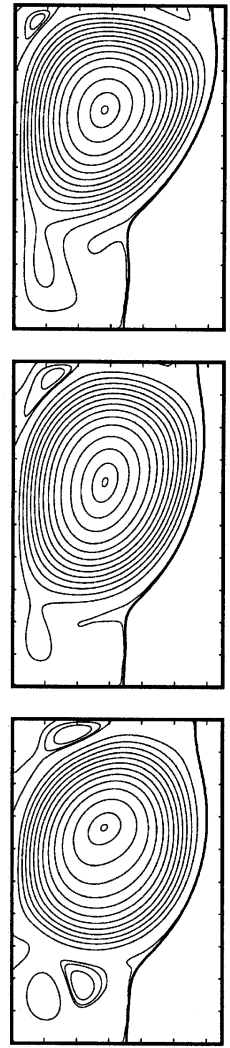

400
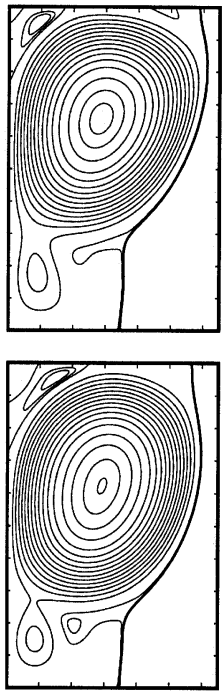

NOT

AVAILABLE

Figure 4. Streamlines and interface at time 2,000s for three grids and three discretization schemes.

as sound as it looks, is nevertheless seriously contradicted by the results obtained by the centered scheme at time $550 \mathrm{~s}$. As may be seen in Figure $3 a$, three rolls are displayed by the $100 \times 100$ grid solution, while only two are displayed by each of the $200 \times 200$ and $400 \times 400$ solutions. One has to look at the melting history in order to explain this rather strange result. The $100 \times 100$ grid is too coarse to capture the four-roll structure that prevails early in the melting process (at time $200 \mathrm{~s}$ ). When a roll merging occurs for the $400 \times 400$ grid solution, the locations of the three resulting rolls are not the same as for the $100 \times 100$ grid. Subsequently, the rolls grow at their initial locations. Because the top two rolls are closer in the $400 \times 400$ solution than in the $100 \times 100$ solution, where the rolls are more evenly spaced, the pairing occurs 130 s earlier. A similar explanation holds for the $200 \times 200$ grid result, which captures four rolls only during a short time interval near $t=140 \mathrm{~s}$. This observation has a very important bearing: any initial discrepancy in roll location, due either to a perturbation or to insufficient resolution, will influence the entire fluid flow for a very long time. Therefore, it is very important to capture the initial roll structure with enough resolution. As an example of resulting incorrect behavior, we mention that 
one of our simulations $(100 \times 100$ - centered $)$ resulted in four major roll mergings, while other simulations showed only three roll mergings.

Comparison with earlier results is also necessary in order to draw conclusions. Notice that the coarsest grid used in the present study $(100 \times 100)$ is actually finer than any of the grids used by the tin-melting benchmark contributors [36] who found a single roll structure. Moreover, those contributors also used first-order discretization schemes. Our work shows that a monocellular structure was to be expected from such a combination. The few contributors who used higher-order discretization reported a multicellular structure, although no results with streamlines were published.

A summary sketch of the roll structure obtained for each choice of grid size and discretization scheme as a function of time is shown in Figure 5. Each line on the plot corresponds to one particular combination of scheme and grid. A step change corresponds to a bifurcation or a roll merging. The number of rolls prevailing during a particular time interval (between two consecutive steps) is indicated by a corresponding number of small circles. Usually, the number of rolls decreases by one after a roll merging. An exception to that is the third roll merging; there are two rolls, both before and after the merging.

Notice that at any given time the number of rolls in the cavity is larger for finer grids and higher-order discretization schemes (except for the $100 \times 100$ centered solution that was mentioned earlier in this section). The number of rolls is roughly the same for the hybrid and centered schemes when the $400 \times 400$ grid is being used. Therefore, one may conclude that the numerical solution is close to converged and that the expected pattern is: one roll early, four rolls after time $120 \mathrm{~s}$, three rolls after time $220 \mathrm{~s}$, two rolls after time $460 \mathrm{~s}$, then merging at time $1,050 \mathrm{~s}$, resulting in the same number of rolls, two. The number of rolls decreases afterwards to one in a continuous way.

Both the upwind scheme with a $100 \times 100$ grid and the hybrid scheme with a $50 \times 50$ grid show only one roll during the entire numerical simulation. This is in accordance with earlier results of tin melting as well as gallium melting, where coarse

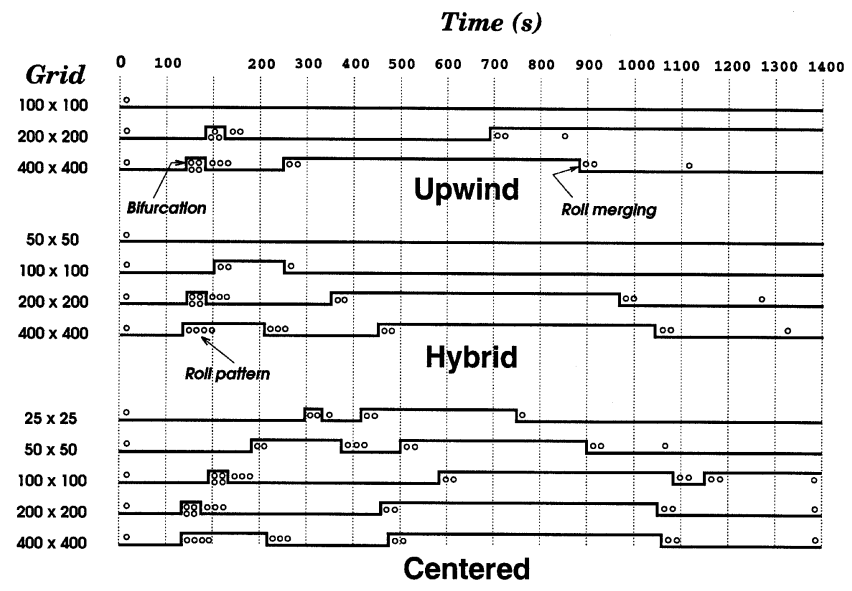

Figure 5. Roll structure as a function of time for three discretization schemes and five grid sizes. 
grids (from $15 \times 15$ to $80 \times 80$ ) and overall first-order schemes were used. Among the 13 contributors of the tin benchmark problem [36], nine participants fall in this category.

The centered scheme has often been criticized for being nonmonotone, generating oscillations, and being prone to numerical unstability. This fact was an additional argument in favor of those who found a one-cell structure for the gallium melting problem and whose results agreed quite well with experiments. As the present work shows, even the upwind scheme captures the four rolls early in the melting process with a sufficiently fine grid $(400 \times 400)$. This eliminates any doubt about the validity of the numerical solution and the multicellular nature of the flow.

In conclusion, the solution of the mathematical model selected for the numerical simulation of the tin melting problem is expected to be multicellular. Further refinement is needed in order to obtain a grid-converged solution.

\subsection{Solid-Liquid Interface}

The shape and location of the solid-liquid interface at several times during the melting process is provided in Figure 6 . Results are provided for three discretization schemes and five grids $(25 \times 25,50 \times 50,100 \times 100,200 \times 200$, and $400 \times 400)$. Each

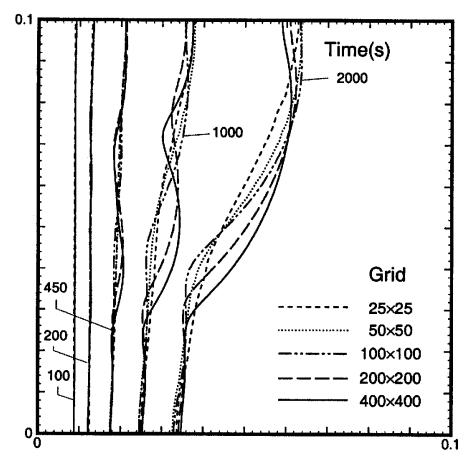

(a) Upwind

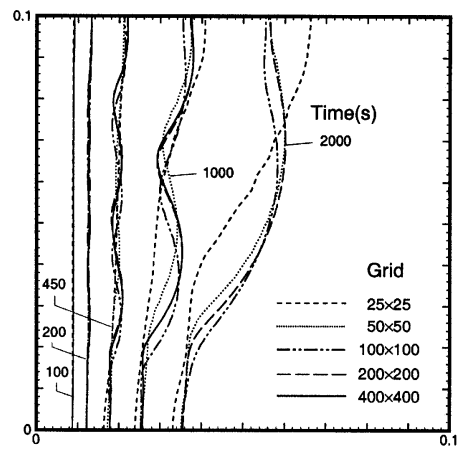

(c) Centered

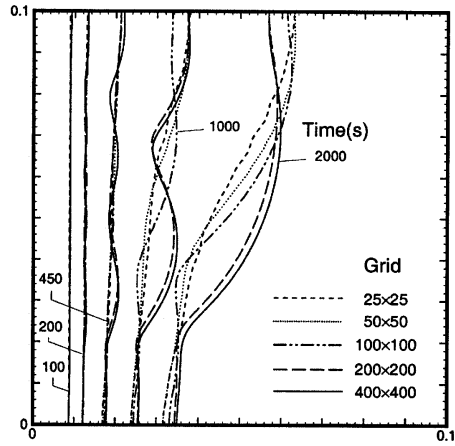

(b) Hybrid

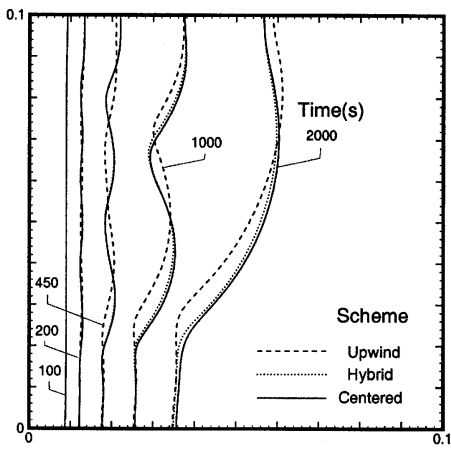

(d) All schemes

Figure 6. Solid-liquid interface for three schemes and several grid sizes. 
of the first three subfigures, $6 a-6 c$, corresponds to the results for one particular scheme. As melting proceeds, the solid-liquid interface moves to the right.

At time $100 \mathrm{~s}$, the interface is flat and identical for all schemes and all grids. This is due to the fact that convection is still weak, and the problem is driven by heat conduction, rendering all schemes equivalent, centered differencing being used for the diffusion terms by all of them. At time $200 \mathrm{~s}$, the interface becomes wavy, due to faster melting near the roll locations. The number of bumps reflects the number of rolls in the melt. As melting proceeds, the depth of the troughs increases, resulting in larger discrepancies among the interface location obtained by different schemes and grids. A $15 \%$ discrepancy between coarse and fine grid results may be noticed at time $450 \mathrm{~s}$ for both hybrid and upwind schemes.

At time $1,000 \mathrm{~s}$, find-grid $(400 \times 400)$ solutions exhibit a two-bump interface, while low-accuracy solutions (upwind $-25 \times 25$ to $100 \times 100$, hybrid $-25 \times 25$ to $100 \times 100$, and centered $-25 \times 25$ ) show only one bump. In their second publication [36], the contributors to the tin melting exercise were divided into two groups. In the first group (the larger), contributors found a one-bump interface, while in the second group, a two-bump interface was reported. Contributors in the first group used lower-order schemes and coarse grids (less than $100 \times 100$ ), while in the second group, grids were finer and discretization schemes of higher order. Hence, one can conclude from the present results that the single-bump interface results from insufficient numerical resolution.

As shown on the plots at time 2,000 s, faster melting occurs at the bottom for finer grids and more accurate schemes. Hence, even at later times in the melting process, interface shape is strongly affected by the grid size and the roll pattern in the flow. This contradicts the results of the tin-melting benchmark exercise contributors [36], who indicated rather good agreement among all the solutions at time 2,500 s. The unavailability of most accurate numerical solutions at time $2,500 \mathrm{~s}$, as well as the use of large time steps by some contributors, could explain the results in [36].

To allow a comparison among the three schemes, the solid-liquid interface obtained with a $400 \times 400$ grid at various times during the melting process is sketched in Figure $6 d$ (at time 2,000 s, the results for the centered scheme are from a $300 \times 300$ grid, due to the unavailability of the $400 \times 400$-grid solution). Notice the very good agreement between the results for hybrid and centered schemes. This fact is generally a good indicator that the solution is close to converged.

\subsection{Nusselt Number Plots}

The average Nusselt number, $\mathrm{Nu}$, at the hot wall is a good indicator of how convection affects overall conduction through the cavity. A correlation for $\mathrm{Nu}$, derived in [36], is used for comparison purposes. $\mathrm{Nu}$ results from three grids and three schemes are shown in Figure 7. For both upwind and hybrid schemes, the $100 \times 100$-grid solution agrees quite well with the correlation, a very smooth curve. As the grid is refined, $\mathrm{Nu}$ assumes values larger than those of the correlation. In addition, sudden drops of the $\mathrm{Nu}$ plot appear at times corresponding to roll pairings. The drops are more pronounced for finer grids and higher-order schemes. More accurate solutions also exhibit oscillations prior and after roll mergings. The highfrequency oscillations shown on the centered plot after time $1,300 \mathrm{~s}$ are possibly of 


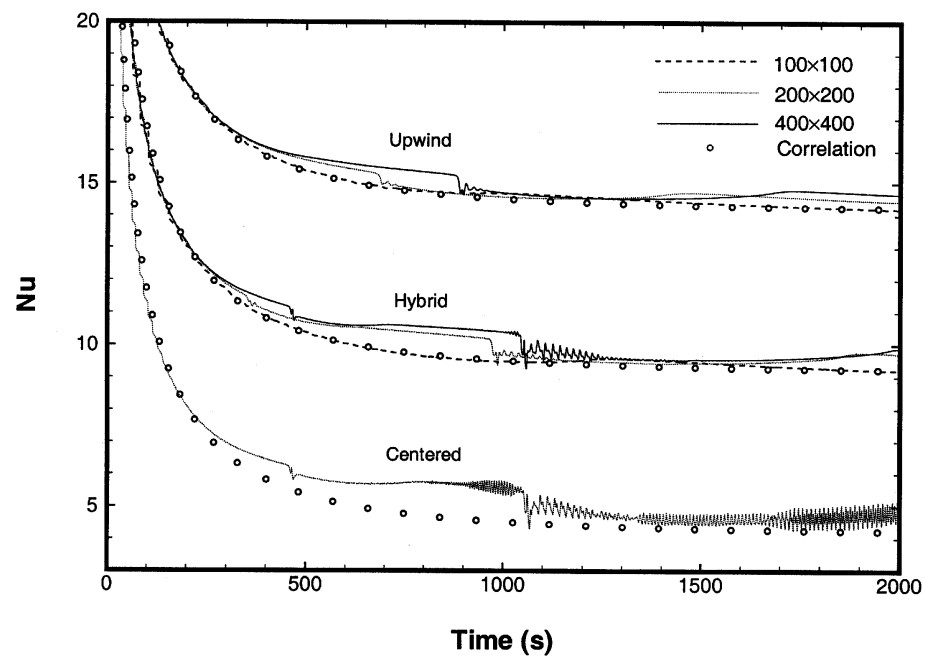

Figure 7. Average Nusselt number at the hot wall as a function of time for three schemes and three grids.

numerical origin, due to the use of a nonmonotone, centered scheme. Further investigation is necessary in order to check this assertion. The centered, $100 \times 100$, and $400 \times 400$ results are not shown in the figure, being very similar to the $200 \times 200$ solution.

\subsection{Volume of Melt}

From an engineering point of view, the rate of melting is one of the most important parameters of the problem. The time evolution of the total liquid fraction in the cavity (ratio of volume of melt to volume of cavity) is a factor that has been widely used as a monitoring parameter in earlier publications. From the liquid fractionversus-time plot, one can get both the rate of melting (slope of the tangent line at a given time) and the average melting rate (ratio of current liquid fraction and time).

Figure 8 displays the time evolution of the total fraction of liquid in the cavity. The effect of grid-refinement is shown in Figure $8 a$ for the hybrid scheme. It may be noticed that the liquid fraction increases as the grid is refined. This is also true for the upwind and centered schemes. A comparison among the three discretization schemes is also shown in Figure $8 b$ for the $200 \times 200$ grid. Again, the liquid fraction is seen to increase as the discretization scheme order is increased. Therefore, one may conclude that more accurate numerical solutions are expected to return higher melting rates, an expected result since convection is stronger for these solutions.

The value of the total liquid fraction in the cavity at time 2,500 s obtained in the present study with the $200 \times 200$ - centered simulation is about 0.6 , a value $5 \%$ larger than the largest prediction from the tin-melting benchmark exercise contributors [36].

Contrary to the Nusselt-number plot, the liquid fraction-versus-time plot is a very smooth curve, free of oscillations, sudden drops, and peaks. This is understood, since the melting time scale is much larger than the convection time scale. 


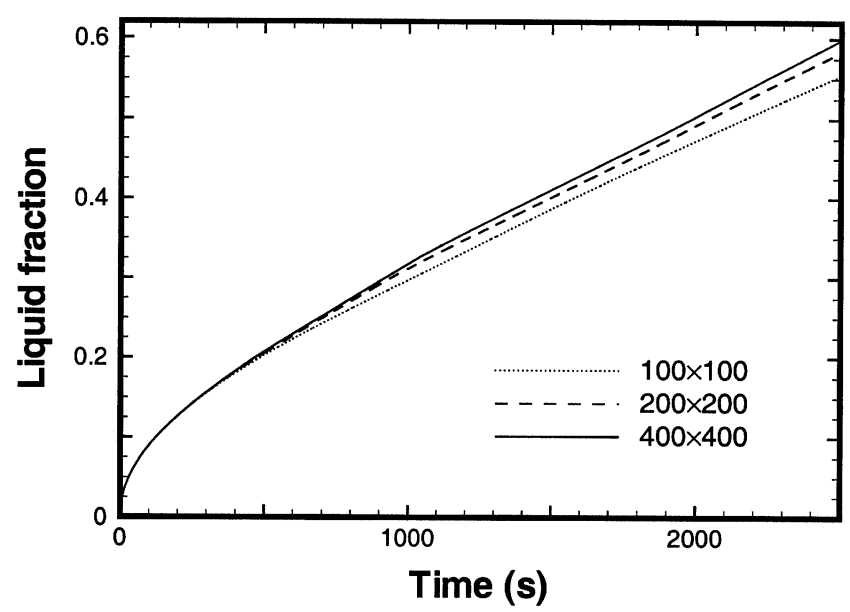

(a) Hybrid scheme

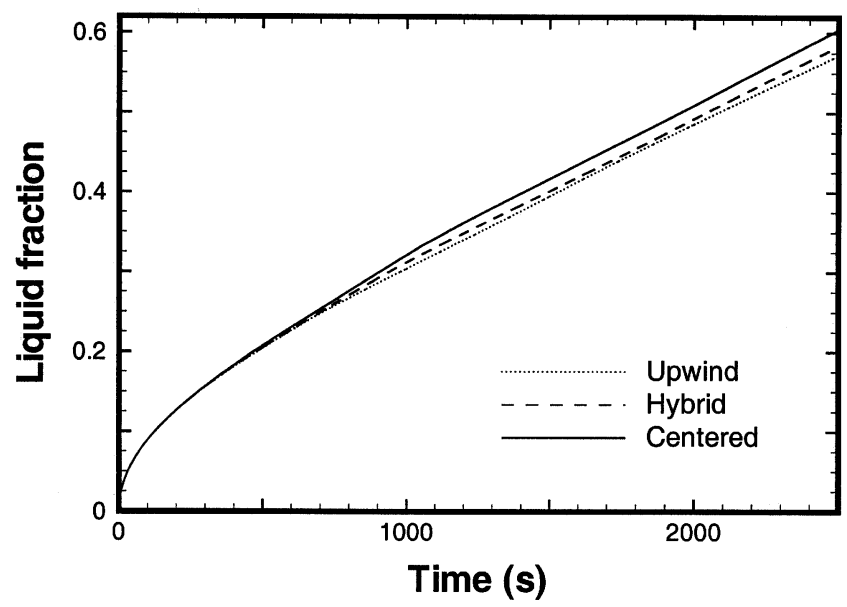

(b) $200 \times 200$ grid

Figure 8. Time evolution of the total fraction of liquid in the cavity.

\subsection{Convergence Plots}

A comparison among three schemes is provided in Figure 9 for the convergence pattern under grid refinement. Two parameters are considered: the minimum value of the streamfunction in the melt, $\psi_{\min }$, and the total liquid fraction in the cavity. The abscissa shows to the number of nodes in one direction (same number in both directions). For all schemes, the solutions converge toward a common value. The centered solution tends to converge faster than the hybrid and upwind solutions; 200 nodes are enough to get an accurate solution at time $1,000 \mathrm{~s}$, while 300 nodes are needed at time $200 \mathrm{~s}$, earlier in the melting process. 


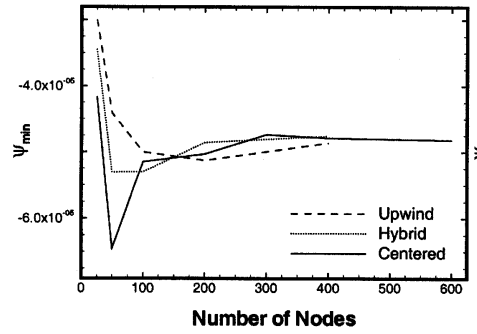

(a) Time $200 s$

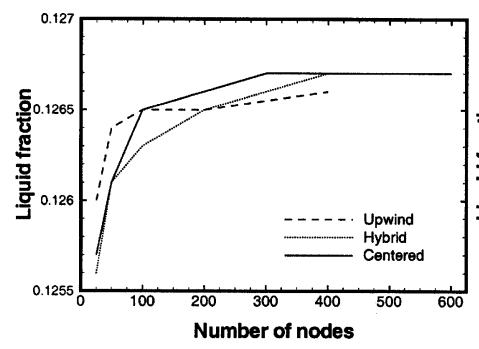

(c) Time 200s

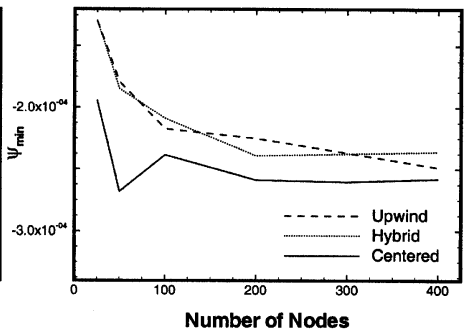

(b) Time $1000 \mathrm{~s}$

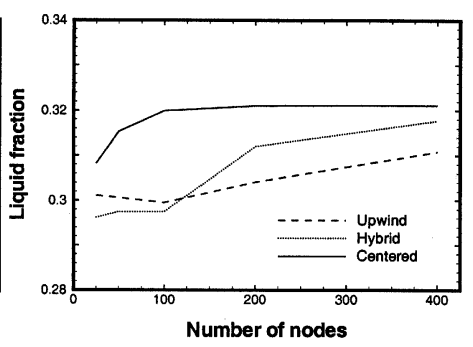

(d) Time $1000 \mathrm{~s}$

Figure 9. Convergence of minimum streamfunction value and cavity liquid fraction under grid refinement.

\section{RESULTS FOR GALLIUM}

In order to check the accuracy of the numerical solution for the gallium melting problem, a grid-refinement study is presented first. All simulations were done with the centered discretization scheme. Figure 10 displays the streamlines and solidliquid interface at time $32 \mathrm{~s}$ obtained with several grids. As may be seen, the results with the two finest grids, $840 \times 600$ and $1,120 \times 800$, agree quite well. Consequently, we present results obtained with the $840 \times 600$ grid. Notice that the grid used for gallium is finer than the grids used for tin. This is necessary because Ra is larger and more cells are to be captured.

Figure 11 shows the time evolution of the flow pattern (streamlines) and solidliquid interface. The results are from an $840 \times 600$ uniform grid, except for the plot at time $280 \mathrm{~s}$, which is from a $560 \times 400$-grid calculation. However, the initial discrepancy between the two grids is very small, and the picture is believed to be very close to the exact solution.

It is interesting that the streamlines obtained in the present work show only four rolls, and a not very well formed and unstable five-roll structure at some point early in the melting process. Our solution is in excellent agreement with the results of Stella et al. [19] at time $32 \mathrm{~s}$. Dantzig [3] reported a roll structure with six rolls. Our grid-refinement study indicates that the roll structure of Stella et al. seems to be correct. Moreover, the very coarse grid used by Dantzig makes the six-roll pattern shown in his plots questionable. The use of two nonmonotone schemes, for space and time, in combination with a very coarse grid, could explain Dantzig's results. 

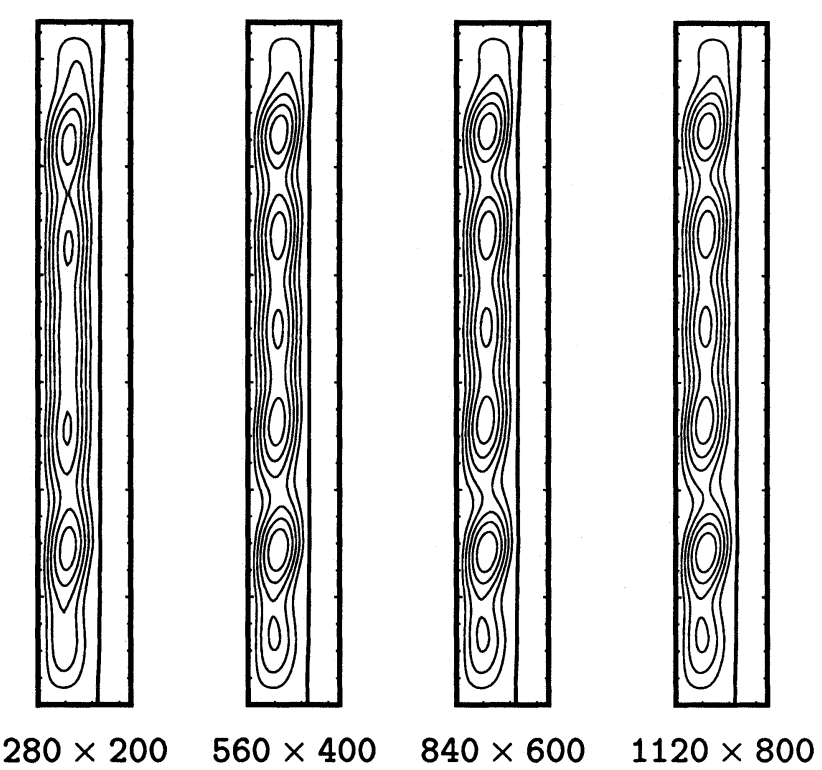

Figure 10. Gallium melting: streamlines and interface at time $32 \mathrm{~s}$ for four grids.

Later, the number of rolls decreases through a process of roll merging, always involving the top two rolls. The present results at time $42 \mathrm{~s}, 85 \mathrm{~s}$, and $155 \mathrm{~s}$ agree very well with those of Stella et al. Interestingly, the selection of plot output times was made on the sole basis of inspecting our results. These times correspond to roll mergings. Those times turned out to be the same as the ones used by Stella et al. for
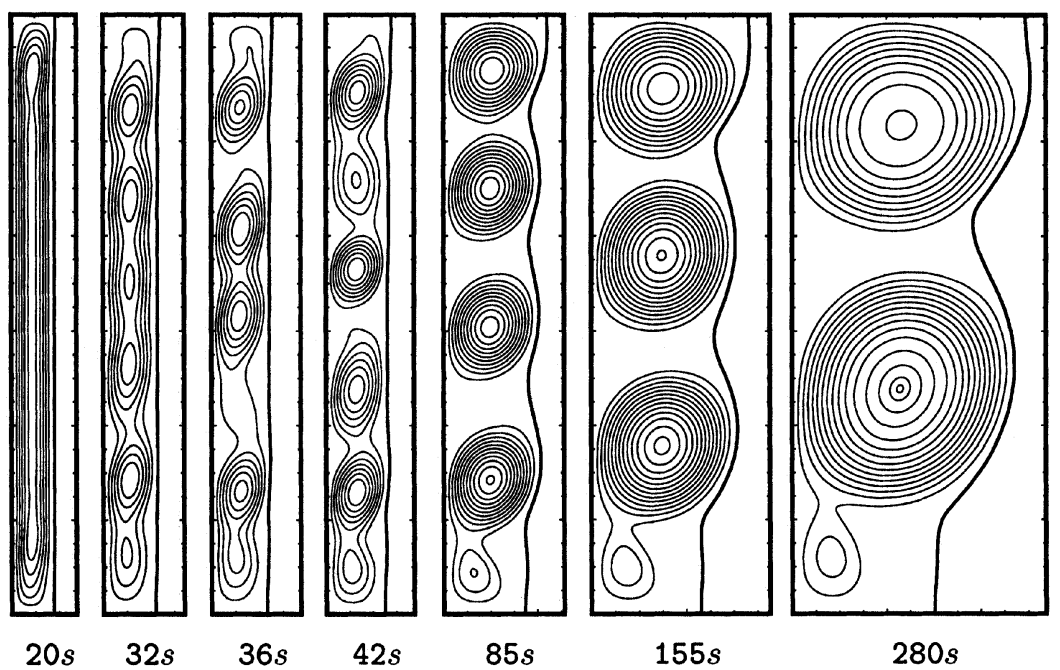

Figure 11. Gallium melting: streamlines and interface at several times during the melting process. 
their output. This underscores the strong agreement between our results and those of Stella et al.

\section{DISCUSSION}

\subsection{Why Use Coarse Grids and Lower-Order Schemes?}

Most earlier studies of gallium and tin melting were done with coarse grids, due to the limitations of computer capabilities. Many authors [17, 28, 30] actually acknowledged that their choice of grid size was a compromise between numerical accuracy and cost of computations. Even on the computers of today, the simulations are highly demanding in CPU time, due to the multiscale nature of the problem. In the present study, the $200 \times 200$-grid simulation up to time $2,500 \mathrm{~s}$ required 2,400 CPU h, 111 runs (restarts), and 3 months of calculations on a Compaq Alpha (667 MHz, ev67) processor. Checking the solution of the gallium melting problem up to time $32 \mathrm{~s}$ with the $1,120 \times 800$ grid has required about $980 \mathrm{CPU}$ hours.

Similarly, lower-order discretization schemes are usually preferred due to their simplicity and monotonicity. Moreover, linear system solvers converge faster on the resulting matrices. Second-order schemes have the drawback of being unstable (oscillatory). However, alternative treatments such as TVD and ENO schemes could be considered [38].

\subsection{Disagreement between Numerics and Experiments: A Tentative Explanation}

As mentioned in the Introduction, Cerimele et al. [20] suggested an interesting idea to explain the disagreement between experimental and numerical results for the gallium melting problem. They argued that the pour-in/pour-out procedure employed by Gau et al. [27] in their experiments to perform measurements was actually leading to a problem different from the one considered in numerical simulations. The experimental procedure involves a fluid restart from rest and from uniform temperature, whereas in the numerical simulations, no such restart is considered. To support their argument, Cerimele et al. performed two calculations, one consistent with the experimental procedure, and one consistent with earlier numerical studies. For the situation consistent with past numerical simulations, Cerimele et al. obtained a very irregular and distorted interface that does not agree with experimental results, and consequently attributed the discrepancies to the inadequacy of the numerical assumptions. However, if one looks more closely at the interface shape they obtained, it appears that this interface shape does not agree with earlier numerical studies either. The irregular interface shape they obtained is very likely due to a numerical instability resulting from insufficient convergence of energy; we observed similar erroneous results in some of our simulations. Moreover, the results of Cerimele et al. for the simulation corresponding to the experimental procedure actually do not improve on past numerical studies, since they also show a two-bump interface as in Dantzig [3], which indicates that their results do not agree with experiments. Consequently, it does not seem that a positive result has been obtained for the argument invoked in their study. 


\subsection{The Myth of Grid-Converged Solution}

A closer look at past published works about the gallium and tin melting problems shows that most authors claimed their solution was grid-converged. A large number of authors [2, 4-7, 10-12, 29-33] did not report results for grid refinement and contented themselves with assertions such as "Further refinements in grid or time step did not produce discernible improvements," "After conducting a grid refinement study, a $42 \times 32$ grid was chosen," or "Similar results were obtained with a $51 \times 51$ grid." Very few authors actually presented grid-refinement results. Viswanath et al. [7] showed the evolution of the volume of melt as the grid was refined from $30 \times 20$ to $50 \times 40$ and concluded that a change in $3 \%$ was acceptable. Rady et al. [12] presented the evolution of the extrema of streamfunction, Nusselt number, and volume of melt values for grids ranging from $20 \times 20$ to $35 \times 35$.

Although most authors in earlier studies have argued that their numerical solutions were grid-converged or close to grid-converged, it is clear from the present study that the single-roll solution they obtained is not grid-converged. Since most earlier studies used the upwind discretization scheme, or a scheme such as hybrid and power law, which reverts to upwind for high values of the Peclet number, an explanation to this anomaly may be provided by the comment of Leonard [39] about low-order schemes:

The convergence rate of such methods is usually so anomalously slow ... in some grid refinement studies..., that in the absence of other solution or experimental data, one can easily convince oneself that a "grid-converged solution" has been reached (to some level of accuracy). (p. 54)

This shows that tougher measures are necessary in order to check the accuracy of numerical solutions for this kind of problem.

\subsection{Too Coarse Grids Early in the Melting Process}

The results presented in our study have shown that the number of rolls early in the melting process needs to be correct in order for the numerical solution to be accurate later. It was shown that a rather coarse grid can capture the correct number of rolls at later times, due to the fact that the rolls grow larger. Later, however, the locations of these rolls would be incorrect. For example, three rolls are obtained with the $100 \times 100$ and the $400 \times 400$ grids (and the centered scheme) at time $450 \mathrm{~s}$, but their locations are different. As a result, merging of the top two rolls happens $130 \mathrm{~s}$ earlier for the $400 \times 400$ grid. A typical result in this category is the one presented by Cruchaga et al. [24] for the gallium melting problem. Due to too coarse a grid, Cruchaga et al. captured only two rolls early in the melting process. Later, the number of rolls increased to three.

Another issue related to using too coarse grids early along with the centered scheme is the problem of flow unstabililty. In our results with a $50 \times 50$ grid and the centered discretization scheme, we noticed large overshoots and undershoots for the extrema of streamfunction values (about $200 \%$ ) as well as an unclear roll structure, constantly evolving. It was actually difficult to decide on a number of rolls and the roll locations (flat shape). This phenomenon could explain the failure of Dantzig [3] 
to obtain results for coarse grids. Dantzig reported that his code could not converge when coarse grids were employed. His use of a nonmonotone second-order discretization scheme for time may have worsened the situation.

\subsection{Validation versus Verification}

The existence of a controversy for gallium and tin melting problems is due partly to confusion between two very important concepts known as verification and validation (Roache [40]). Verification amounts to checking that a computer code designed for solving a given set of partial differential equations (model) is free of any bug. This is achieved through testing on problems with known solutions, performing grid-refinement studies, and ensuring that the convergence pattern is consistent with the order of the discretization scheme. Validation, on the other hand, is concerned with the adequacy of a mathematical model for a particular physical application. Validation is performed by comparing a verified numerical solution to the selected mathematical model with physical observations.

For the problem at hand, gallium or tin melting, the correct numerical solution is the one that represents the converged solution for the mathematical model selected. It may or not agree with experimental observation. On the other hand, a nonconverged numerical solution is unacceptable even if it agrees with experimental observation, since it is not a correct solution to the mathematical model selected.

Consequently, multicellular flow pattern is the correct answer to the numerical simulations, while further investigations will be necessary in order to determine whether the model is inadequate, or previous experimental studies were not consistent with the assumptions made in the numerical simulations.

\subsection{Checking Accuracy without a Reference Solution}

In the absence of a reference solution (benchmark solution or analytical solution), two procedures have been commonly employed to assess the accuracy of numerical results for phase change involving convection: comparison with experimental results and comparison with other numerical results. However, neither procedure is a valid method to assess accuracy. Direct comparison with experiments bypasses the verification and validation processes, while comparison with another numerical result is valid only if that result has itself been assessed. Arguments of this type have been used in [14] to support the single-cell solution.

A reference solution could actually be obtained by a simple grid-convergence study. It is a simple computational exercise that does not require any experimental check. The numerical solution obtained would be a true reference solution for the selected mathematical model, and researches would be able to use it to verify their own codes.

\subsection{Is the Multicellular Solution Physically Correct?}

Since the muticellular pattern in the gallium and tin melting problems has not been reported experimentally, it is legitimate to question its validity. A multicellular pattern has indeed been reported in the experimental work of Chikhaoui et al. [41], 
as indicated by Dantzig [3]. Chikhaoui et al. found six cells in an experiment involving natural convection of air in a vertical slot. Involving a melting time scale much larger than the convection time scale, the problems of gallium and tin melting may actually be compared to pure natural convection in a vertical slot with vertical boundaries corresponding to the left wall and the solid-liquid interface. In a separate experimental study involving natural convection in a vertical differentially heated cavity with large aspect ratio, Vest and Arpaci [42] observed nine cells. Later the problem was simulated numerically by Leonard et al. [38], who obtained the same roll pattern with a $3 \%$ error. Hence, one may conclude that the possibility of a multicellular flow structure for the problems of tin and gallium melting is viable.

\subsection{What Should Be Done Next}

The present contribution has shown clearly that the solution to the mathematical model commonly adopted for the numerical simulation of the tin and gallium melting problems is multicellular. This does not agree with published experimental observations. The various ideas linked to the problem have been reviewed and clarified. Here, two possible directions are suggested for future work: checking the mathematical model and checking the experimental procedure.

If the experimental results turn out to be correct (single cell), then the mathematical model will have to be modified. Several assumptions may be questioned:

1. The situation is two-dimensional.

2. Thermophysical properties are constant (independent of temperature).

3. Thermal conductivity of solid gallium is isotropic.

4. Thermophysical properties of solid and liquid are equal.

5. Melting does not involve expansion.

6. Other effects, such as surface tension, are neglected.

Among these possibly inappropriate assumptions, two deserve particular attention. Assumption 5 is one that can lead to important changes. Gallium actually shrinks upon melting, by about $3.8 \%$. Therefore, a free interface (gas-liquid) would result upon melting, and surface tension effects might have to be included in the model. On the other hand, three-dimensional effects would result if filling in of liquid gallium was allowed to compensate for volume loss. Also, assumption 1 definitely needs closer examination, as the following comment of Frederic and Quiroz [43] suggests:

...two-dimensionality is only an approximation to the actual behavior, which is always three dimensional. Not all the problems of confined natural convection possess a two-dimensional version,.... A three-dimensional assumption being more realistic, can detect phenomena not seen in the two-dimensional version. (p. 1699).

Questioning the validity of the experimental procedure is another alternative that several researchers $[19,35,36]$ have supported. Do the experiments meet the assumptions of the physical problem? In particular, it is interesting to evaluate to which level of compliance the following are met: 
1. Boundary conditions (isothermal and adiabatic walls)

2. Initial conditions (sudden increase of temperature)

3. Purity of gallium

4. Two-dimensional assumption (is the cavity wide enough?)

Two additional problems associated with experiments are the difficulties in performing three-dimensional measurements and the visualization of streamlines in an opaque melt.

\section{CONCLUSION}

In the present study, numerical simulations for tin and gallium melting in a rectangular cavity heated from a vertical wall have been performed. A thorough comparison among three common discretization schemes and several grid sizes has been presented. A lengthy discussion focusing on the controversy regarding the flow structure and related problems has been provided.

The present contribution has shown that the issues of grid size and discretization scheme, both suggested as an explanation to the discrepancies in earlier results, are actually a problem of convergence of the numerical solution. Earlier arguments suggested to explain discrepancies between experiments and numerical solutions could be termed simply "improper convergence of the numerical solution," and a reference solution can indeed be obtained regardless of agreement between experiments and numerics. It was shown that as the solution converges, the flow structure becomes multicellular. Hence the solution to the mathematical model selected for the numerical simulation is multicellular and earlier results with one single cell are erroneous, due to insufficient grid convergence. The controversy is now easily explained; since the solution of the selected mathematical model does not agree with experimental results, either the mathematical model is inadequate or the experimental procedure did not meet the assumptions of the mathematical model; Future directions include checking earlier experiments by overcoming experimental difficulties or finding a more appropriate model to the problems of tin and gallium melting.

\section{REFERENCES}

1. V. Alexiades and A. D. Solomon, Mathematical Modeling of Melting and Freezing Processes, Hemisphere, Washington, DC, 1993.

2. A. D. Brent, V. R. Voller, and K. J. Reid, Enthalpy-Porosity Technique for Modeling Convection-Diffusion Phase Change: Application to the Melting of a Pure Metal, Numer. Heat Transfer, vol. 13, pp. 297-318, 1988.

3. J. A. Dantzig, Modelling Liquid-Solid Phase Changes with Melt Convection, Int. J. Numer. Meth. Eng., vol. 28 pp. 1769-1785, 1989.

4. M. Lacroix and V. R. Voller, Finite Difference Solutions of Solidification Phase Change Problems: Transformed versus Fixed Grids, Numer. Heat Transfer B, vol. 17, pp. 25-41, 1990.

5. D. Gobin and C. Benard, Influence of the Prandtl Number on the Melting of Metals, in Y. Bayazitoglu (ed.), Fundamentals of Phase Change: Freezing, Melting, and Sublimation, ASME HTD-Vol. 143, pp. 75-81, 1990. 
6. C. P. Desai and K. Vafai, A United Examination of the Melting Process within a TwoDimensional Rectangular Cavity, J. Heat Transfer, vol. 115, pp. 1072-1075, 1993.

7. R. Viswanath and Y. Jaluria, A Comparison of Different Solution Methodologies for Melting and Solidification Problems in Enclosures, Numer. Heat Transfer, vol. 24, pp. $77-$ $105,1993$.

8. C.-H. Kuo and W. C. Schreiber, An Interface-Tracking Method for Solving Substance Phase-Change Problems Using Nonsteady Curvilinear Coordinates, in K. Vafai and J. L. Chen (eds.), Numerical Simulation of Flow and Heat Transfer, ASME HTD-vol. 275, pp. 57-64, 1994.

9. D. J. McDaniel and N. Zabaras, A Least-Squares Front-Tracking Finite-Element Method Analysis of Phase Change with Natural Convection, Int. J. Numer. Meth. Eng., vol. 37, pp. 2755-2777, 1994.

10. W. Shyy, Computational Modeling for Fluid Flow and Interfacial Transport, pp. 430-432, Elsevier, Amsterdam, The Netherlands, 1994.

11. W. Shyy and M. M. Rao, Enthalpy Based Formulation for Phase Change Problems with Application to g-Jitter, Microgravity Science and Technology-VII, vol. 1, pp. 41-49, 1994.

12. A. Rady and A. K. Mohanty, Natural Convection during Melting and Solidification of Pure Metals in a Cavity, Numer. Heat Transfer A, vol. 29, pp. 49-63, 1996.

13. W. Shyy, H. S. Udaykumar, M. M. Rao, and R. W. Smith, Computational Fluid Dynamics with Moving Boundaries, pp. 250-259, Taylor \& Francis, Washington D. C., 1996.

14. V. R. Voller, An Overview of Numerical Methods for Solving Phase Change Problem, in W. J. Minkowycz and E. Sparrow (eds.), Advances in Numerical Heat Transfer, vol. 1, pp. 340-380, Taylor \& Francis, Washington D. C., 1997.

15. W. Shyy, S. Pal, H. S. Udaykumar, and D. Choi, Structured Moving Grid and Geometric Conservation Laws for Fluid Flow Computation, Numer. Heat Transfer A, vol. 34, pp. 369-397, 1998.

16. S. A. Khashan, An Advanced Numerical Model for Phase Change Problems in Complicated Geometries, Ph.D. dissertation, University of Pittsburgh, Pittsburgh, PA, 1998.

17. R. Sampath and N. Zabaras, An Object Oriented Implementation of a Front Tracking Finite Element Method for Directional Solidification Processes, Int. J. Numer. Meth. Eng., vol. 44, pp. 1227-1265, 1999.

18. Y. Jaluria, Numerical Modeling of Thermal Materials Processing Systems, in D. Stock (ed.), Proc. AMEFED-vol. 250, pp. 137-148, 1999.

19. F. Stella and M. Giangi, Melting of a Pure Metal on a Vertical Wall: Numerical Simulation, Numer. Heat Transfer A, vol. 138, pp. 193-208, 2000.

20. M. M. Cerimele, D. Mansutti, and F. Pistella, A Front-Fixing Method for Fluids in Liquid/Solid Phase Change with a Benchmark Test, in European Congress on Computational Methods in Applied Sciences and Engineering, ECCOMAS 2000, Barcelona, 11-14 September 2000.

21. M. A. Cruchaga and D. J. Celentano, A Finite-Element Thermally Coupled Flow Formulation for Phase-Change Problems, Int. J. Numer. Meth. Eng., vol. 34, pp. 279-305, 2000.

22. Y. Jaluria, Fluid Flow Phenomena in Materials Processing-The 2000 Freeman Scholar Lecture, J. Fluids Eng., vol. 123, pp. 173-210, 2001.

23. S. Kim, S. Anghaie, and G. Chen, A Fixed-Grid Two-Phase Numerical Model for Convection-Dominated Melting and Solidification, Proc. First MIT Conf. on Computational Fluid and Solid Mechanics, MIT, Cambridge, MA, 12-14 June 2001.

24. M. A. Cruchaga and D. J. Celentano, A Fixed-Mesh Finite Element Thermally Coupled Flow Formulation for the Numerical Analysis of Melting Processes, Int. J. Numer. Meth. Eng., vol. 51, pp. 1231-1258, 2001. 
25. I. Wintruff and C. Gunther, An Adaptive Moving Grid Model for Convective Melting and Solidification Problems, in P. Ehrhard, D. S. Riley, and P. H. Steen (eds.), Interactive Dynamics of Convection and Solidification, pp. 235-242, Kluwer, Dordrecht, The Netherlands, 2001.

26. T. A. Campbell and J. N. Koster, Visualization of Liquid-Solid Interface Morphologies, J. Crystal Growth, vol. 140, pp. 414-425, 1994.

27. C. Gau. and R. Viskanta, Melting and Solidification of a Pure Metal from a Vertical Wall, J. Heat Transfer, vol. 108, pp. 171-174, 1986.

28. B W. Webb and R. Viskanta, Analysis of Heat Transfer during Melting of a Pure Metal from an Isothermal Vertical Wall, Numer. Heat Transfer, vol. 9, pp. 539-558, 1986.

29. M. Lacroix and A. Garon, Numerical Solution Phase Change Problems: and EulerianLagrangian Approach, Numer. Heat Transfer B, vol. 19, pp. 57-78, 1992.

30. F. Wolff and R. Viskanta, Melting of a Pure Metal from a Vertical Wall, Exp. Heat Transfer, vol. 1, pp. 17-30, 1987.

31. C. Benard and D. Gobin, Numerical Simulation of Melting Processes for Metals, in Multiphase Flow, Heat and Mass Transfer, ASMEHTD-vol. 109, pp. 55-60, 1989.

32. C. J. Ho and C. H. Chu, Periodic Melting within a Square Enclosure with an Oscillatory Surface Temperature, Int. J. Heat Mass Transfer, vol. 36, pp. 725-733, 1993.

33. C. J. Ho and C. H. Chu, The Melting Process of Ice from a Vertical Wall with TimePeriodic Temperature Perturbation inside a Rectangular Enclosure, Int. J. Heat Mass Transfer, vol. 36, pp. 3171-3186, 1993.

34. P. Lequere and D. Gobin, Call for contribution, http://m17.limsi.fr/Individu/plq/, 1998.

35. O. Bertrand, B. Binet, H. Combeau, S. Couturier, Y. Delannoy, D. Gobin, M. Lacroix, P. Lequere, M. Medale, J. Mencinger, H. Sadat, and G. Vieira, Melting Driven by Natural Convection. A Comparison Exercise. First Results, Int. J. Thermal Sci., vol. 38, pp. 5-26, 1999.

36. D. Gobin and P. Lequere, Melting from an Isothermal Vertical Wall: Synthesis of a Numerical Comparison Exercise, Comput. Assist. Mech. Eng. Sci., vol. 7, pp. 289-306, 2000.

37. N. Hannoun, A Benchmark Solution for Phase Change with Convection, Ph.D. dissertation, The University of Alabama, Tuscaloosa, AL, 2001.

38. B. P. Leonard and J. E. Drummond, Why You Should Not Use 'Hybrid', 'Power-law' or Related Exponential Schemes for Convective Modelling-There Are Much Better Alternatives, Int. J. Numer. Meth. Fluids, vol. 20, pp. 421-442, 1995.

39. B. P. Leonard, Bounded Higher-Order Upwind Multidimensional Finite-Volume Convection-Diffusion Algorithms, in W. J. Minkowycz and E. M. Sparrow (eds.), Advances in Numerical Heat Transfer, vol. 1, pp. 1-57, Taylor \& Francis, Washington D.C., 1997.

40. P. J. Roache, Verification and Validation in Computational Science and Engineering, chap. 2, Hermosa, Albuquerque, NM, 1998.

41. A. Chikhaoui, P. Bontoux, M. K. Maslanik, and R. L. Sani, Steady Three Dimensional Thermal Convection in a Vertical Rectangular Enclosure: Transition to Multicellular Flow, in M. S. Engelman (ed.), FIDAP User's Conference, pp. 4.1-4.19, Fluid Dynamics International, Evanston, IL, 1987.

42. C. M. Vest and V. Arpaci, Stability of Natural Convection in a Vertical Slot, J. Fluid Mech., vol. 36, pp. 1-15, 1969.

43. R. L. Frederic and F. Quiroz, On the Transition from Conduction to Convection Regime in a Cubical Enclosure with a Partially Heated Wall, Int. J. Heat Mass Transfer, vol. 44, pp. 1699-1709, 2001. 